\title{
Memory and correlation effects in the exciton-phonon kinetics
}

\author{
Gianandrea Mannarini ${ }^{* 1,2}$ and Roland Zimmermann ${ }^{2}$ \\ ${ }^{1}$ National Nanotechnology Laboratory of CNR, via Arnesano 16, 73100 Lecce, Italy \\ ${ }^{2}$ Institut für Physik der Humboldt-Universität zu Berlin, Newtonstr. 15, 12489 Berlin, Germany
}

Received 19 February 2006, revised 29 March 2006, accepted ?

Published online?

Key words memory, correlation, non-Markov, exciton, phonon.

PACS 78.20.-e, 63.20.Ls, 73.43.Cd

Memory and correlation effects in the interband absorption from quantum wells due to exciton-phonon dynamics are investigated. They are traced back to the frequency dependence and matrix character of the self energy arising in a 2nd Born theory. It is found that interpeak absorption increases with respect to the case in which this memory and correlation effects are neglected.

Copyright line will be provided by the publisher

The interaction between excitonic and phonon modes in semiconductor nanostructures leads to both relaxation of the excitonic population as well as to pure dephasing of the optical polarization. In small quantum dots, where the interstate relaxation is tiny, clear signatures of pure dephasing effects have been measured, see e.g. the extensive work done in [1] and [2]. In this case, an accurate theoretical modeling is possible in the framework of an extended version of the Independent Boson Model [3]. In quantum wells (QW), disorder-localized exciton states have significant spatial overlap and can be spectrally rather close, thus a combination of population relaxation and pure dephasing is expected. However, as a consequence of the so-called Markov approximation, the absorption spectrum of a QW results to be a superposition of Lorentzian peaks, thus accounting for population relaxation only. We improve on this situation by going beyond the Markov approximation in the solution of a 2nd Born density-matrix theory for excitons and acoustic phonons in disordered QW.

We start here with the same model which was used in [4]: center-of-mass (COM) excitons are created in disorder eigenstates $|\alpha\rangle$ of the QW by $B_{\alpha}^{\dagger}$ and are coupled to longitudinal acoustic phonons ( $a_{\mathbf{q}}^{\dagger}$ creates a bulk like phonon state $|\mathbf{q}\rangle$ ) via deformation potential interaction:

$$
\mathcal{H}=\sum_{\alpha} \epsilon_{\alpha} B_{\alpha}^{\dagger} B_{\alpha}+\sum_{\mathbf{q}} \hbar w_{\mathbf{q}} a_{\mathbf{q}}^{\dagger} a_{\mathbf{q}}+\sum_{\alpha \beta \mathbf{q}} t_{\alpha \beta}^{\mathbf{q}}\left(a_{\mathbf{q}}^{\dagger}+a_{-\mathbf{q}}\right) B_{\alpha}^{\dagger} B_{\beta} .
$$

The eigenenergies $\epsilon_{\alpha}$ are obtained from a microscopic simulation of the COM exciton in a QW with interfacial disorder [5] and the matrix elements $t_{\alpha \beta}^{\mathbf{q}}$ ensure spatial overlap of the exciton states $|\alpha\rangle$ and $|\beta\rangle$ and the phonon wavefunction $|\mathbf{q}\rangle$. Final output of our theory is the linear absorption spectrum $\alpha(\omega)$, which is connected to the Fourier transform of the exciton polarization $P_{\alpha}(t)=\left\langle B_{\alpha}^{\dagger}(t)\right\rangle$ via

$$
\alpha(\omega)=\operatorname{Im} \sum_{\alpha} m_{\alpha} P_{\alpha}(\omega)
$$

where $m_{\alpha}$ are matrix elements of the dipole coupling to the light field. Both $m_{\alpha}$ and $t_{\alpha \beta}^{\mathbf{q}}$ are given explicitly in [4]. When deriving the equation of motion for $P_{\alpha}(t)$, a hierarchy of equations is obtained in which the polarization couples to expectation values of mixed exciton-phonon operators. Combining two phonon

* Corresponding author: e-mail: Gianandrea.Mannarini@unile.it, Phone: +39 0832 29 8114, Fax: +39 0832298237 
operators into the phonon occupation function $n\left(\omega_{\mathbf{q}}\right)=\left\langle a_{\mathbf{q}}^{\dagger} a_{\mathbf{q}}\right\rangle$ allows one to end up with a differential equation for $P_{\alpha}(t)$ containing a time integration over the past and cross correlations to all other exciton states $\beta \neq \alpha$ :

$$
\left(-i \hbar \partial_{t}-\epsilon_{\alpha}\right) P_{\alpha}(t)=m_{\alpha} \delta(t)+\hbar^{-1} \sum_{\beta} \int d t^{\prime} \Sigma_{\alpha \beta}\left(t-t^{\prime}\right) P_{\beta}\left(t^{\prime}\right) .
$$

Here we have considered an impulsive optical excitation of unit strength at $t=0$. The kernel $\Sigma_{\alpha \beta}(t)$ contains 2 nd powers of the exciton-phonon coupling $t_{\alpha \beta}^{\mathbf{q}}$ : thus, this truncation scheme is called 2nd Born approximation. When memory and correlation are neglected, the kernel reduces to

$$
\begin{aligned}
\Sigma_{\alpha \beta}(t) & \rightarrow \delta_{\alpha \beta} \delta(t)\left[\Delta_{\alpha}+i \frac{\hbar \gamma_{\alpha}^{M}}{2}\right] \\
\gamma_{\alpha}^{M} & =\frac{2 \pi}{\hbar} \sum_{\eta \mathbf{q}}\left|t_{\alpha \eta}^{\mathbf{q}}\right|^{2}\left[n\left(\omega_{\mathbf{q}}\right) \delta\left(\epsilon_{\eta}-\epsilon_{\alpha}-\hbar \omega_{\mathbf{q}}\right)+\left(1+n\left(\omega_{\mathbf{q}}\right)\right) \delta\left(\epsilon_{\eta}-\epsilon_{\alpha}+\hbar \omega_{\mathbf{q}}\right)\right]
\end{aligned}
$$

where $\Delta_{\alpha}$ is a polaron shift (in the following neglected because tiny) and $\gamma_{\alpha}^{M}$ is the Fermi's golden rule scattering rate. The label $M$ reminds that this further simplification is also called Markov approximation. Clearly, this leads to an absorption spectrum which is a superposition of Lorentzian peaks. However, finer structures are found in the spectrum $\alpha(\omega)$ if the Markov approximation Eq. (4) is not carried out. For computing them, we find convenient to Fourier transform Eq. (3), leading to the frequency-domain version of the kernel

$$
\Sigma_{\alpha \beta}(\omega)=\sum_{\eta} \int d E \frac{n(E) J_{\alpha \beta}^{\eta}(E)}{\hbar \omega-i 0^{+}-\epsilon_{\eta}+E} .
$$

The kernel or self energy $\Sigma_{\alpha \beta}(\omega)$ depends on a coupling function $J_{\alpha \beta}^{\eta}(E)$ which decides which exciton states are coupled to the phonon bath,

$$
J_{\alpha \beta}^{\eta}(E)=\operatorname{sgn}(E) \sum_{\mathbf{q}} t_{\alpha \eta}^{\mathbf{q}} t_{\eta \beta}^{-\mathbf{q}} \delta\left(|E|-\hbar \omega_{\mathbf{q}}\right) .
$$

In particular, $J_{\alpha \beta}^{\eta}(E)$ is proportional to the overlap between $|\alpha\rangle$ and $|\eta\rangle$ and between $|\eta\rangle$ and $|\beta\rangle$. The self energy approach leads not only to Lorentzian peaks (or Zero Phonon Lines, ZPLs), but also to satellite absorption bands (broad bands) in $\alpha(\omega)$. However, since $O\left(N^{3}\right)$ components of $J$ have to be computed, this level of approximation is computationally expensive when the number $N$ of exciton states gets large. Further, it would be interesting to have a level of approximation in which the cross-correlations among exciton states predicted from Eq. (3) are neglected, but the memory effects are still accounted for. Thus, we consider also a diagonal (D) non-Markov approximation, which has got a numerical cost $O\left(N^{2}\right)$ but still reproduces both Lorentzian ZPLs and broad bands in the absorption with acceptable accuracy. In this case, the self energy is diagonal, i.e. $\Sigma_{\alpha \beta}^{D}(\omega)=\delta_{\alpha \beta} \Sigma_{\alpha \alpha}(\omega)$. The differences between the spectrum $\alpha_{F}(\omega)$ computed using the full self energy Eq. (5) and the spectrum $\alpha_{D}(\omega)$ obtained within the diagonal approximation are due to off diagonal elements of the Green's function

$$
[G(\omega)]_{\alpha \beta}^{-1}=\left(\hbar \omega-i 0^{+}-\epsilon_{\alpha}\right) \delta_{\alpha \beta}-\Sigma_{\alpha \beta}(\omega),
$$

which vanish within the diagonal non-Markov approximation. Eq. (7) is derived from Fourier transformation of Eq. (3) and by noticing that $P_{\alpha}(\omega)=\sum_{\beta} G_{\alpha \beta}(\omega) m_{\beta}$.

For evaluating the presented theory, we consider a system of 10 exciton COM states obtained from diagonalization of a two-dimensional Schrödinger equation with correlated disorder potential, along the lines described in [5]. The 10 eigenfunctions $|\alpha\rangle$ are used for evaluating the coupling functions $J_{\alpha \beta}^{\eta}(E)$ 


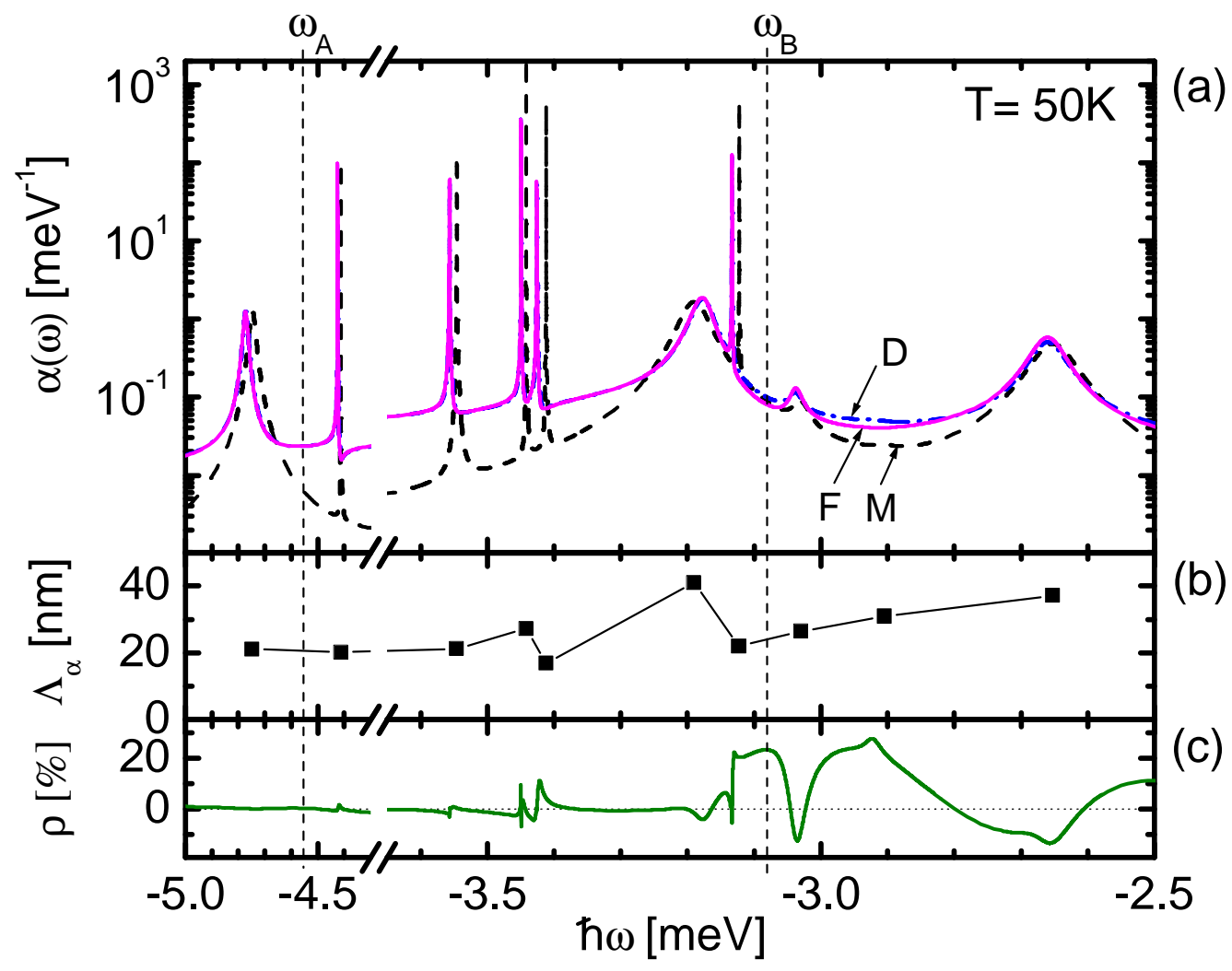

Fig. 1 (Color online) (a) Interband absorption spectrum $\alpha(\omega)$ of 10 exciton states of a $5 \mathrm{~nm} \mathrm{GaAs} / \mathrm{Al}_{0.3} \mathrm{Ga}_{0.7} \mathrm{As}$ QW at a lattice temperature $T=50 \mathrm{~K}$. The full line refers to the full (F) self energy computation, dash-dotted is the diagonal non-Markov (D) approximation, while dashes give the Markov (M) spectrum (superposition of Lorentzians). The 9th peak is not resolved because its oscillator strength $m_{9}$ is vanishing small. (b) Localization lengths $\Lambda_{\alpha}$ of the 10 states calculated as inverse participation ratios. (c) Relative accuracy $\rho=\left(\alpha_{D}-\alpha_{F}\right) / \alpha_{F}$. The vertical cuts refer to the frequencies $\omega_{A}$ and $\omega_{B}$ used in Fig.2]

and, using also the eigenenergies $\epsilon_{\alpha}$, the self energy and the absorption spectra can be calculated. In Fig.11 we compare the Markovian absorption (superposition of Lorentzian peaks) to the spectra obtained using non-Markovian self energies (both D and F version). The most striking feature is the enhancement of the inter-peak absorption with respect to the Markovian case. This effect is more important for states which are spatially less extended, as indicated by the localization lengths $\Lambda_{\alpha}$ displayed in Fig. 10. The formation of the broad bands is mostly due to the frequency dependence in the self energy denominator (cp. Eq. (5)). This frequency dependence is a many-body effect indicating that memory in the exciton-phonon kinetics is not negligible. Since this phenomenon does not need to be related to relaxation among different states (set all indexes equal to $\alpha$ in Eq. (5)), it is also said to be an effect of "pure dephasing". Minor effects present in the non-Markovian spectra (both D and F) are tiny renormalization of the peak positions (polaron shifts) and small dispersive features in the ZPLs: Both features are due to the real part of the self energy. Any residual difference between the $\mathrm{D}$ and $\mathrm{F}$ spectra is due to the cross-correlations predicted from Eq. (3). In Fig.11 we show that they are responsible for deviations up to about $20 \%$ in the numerical values of the broad band absorption, while the ZPLs are mostly correctly accounted for. The physical origin of the correlations is that the self energy approach accounts for scattering events $|\alpha\rangle \rightarrow|\eta\rangle \rightarrow|\beta\rangle$, where a third state $|\eta\rangle$ can "bridge" between initial and final state. Thus, it is not necessary that $|\alpha\rangle$ and $|\beta\rangle$ do overlap for scattering into each other. Due to the $t$ matrix elements appearing in Eq. (6), just $|\alpha\rangle$ and 

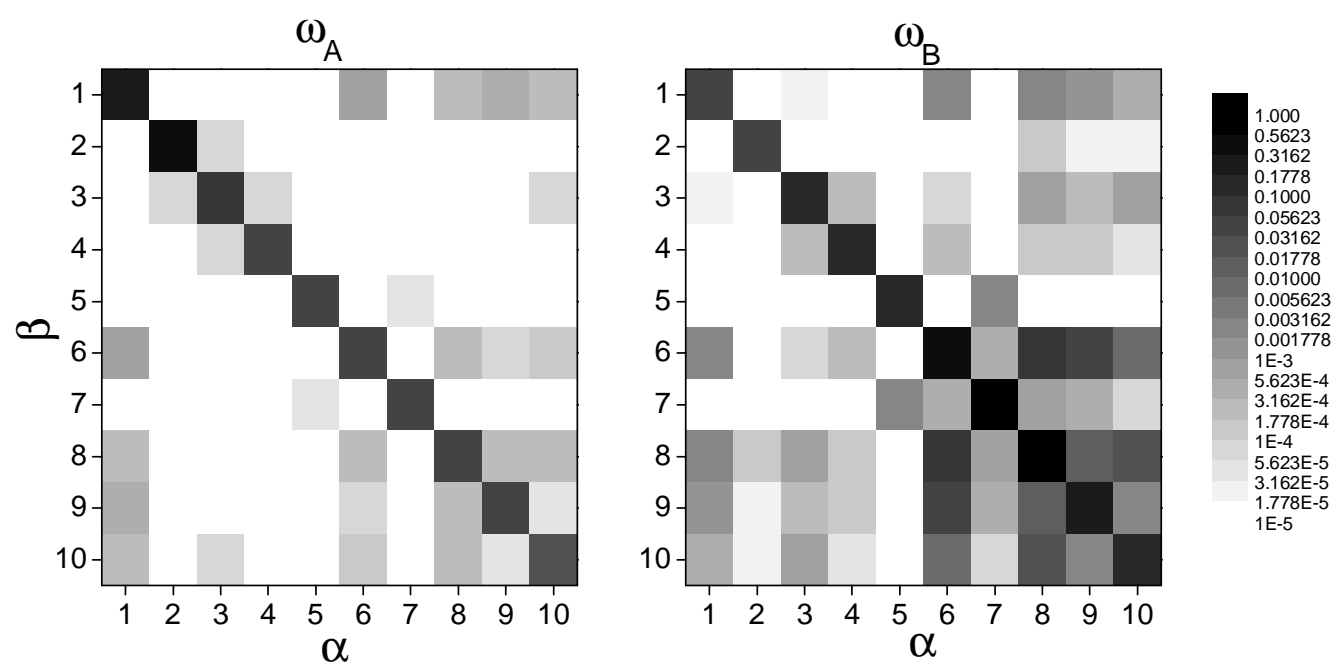

Fig. 2 Green's functions $\left|G_{\alpha \beta}(\omega)\right|$ at frequency $\omega=\omega_{A}$ (left matrix) and $\omega=\omega_{B}$ (right matrix) defined by the cut positions in Fig.1 Logarithmic gray scale over 5 orders of magnitude; values normalized to the maximum of $\left|G_{\alpha \beta}\left(\omega_{B}\right)\right|$. For a strictly diagonal self energy, all off diagonal elements should be zero (white).

$|\eta\rangle$, and $|\eta\rangle$ and $|\beta\rangle$ independently, have to overlap. Conversely, within both diagonal non-Markov and Markov approximation only one step transitions $|\alpha\rangle \rightarrow|\beta\rangle$ are allowed in which $|\alpha\rangle$ and $|\beta\rangle$ directly overlap. A powerful tool for checking which states are correlated is the evaluation of the Green's function matrix $G_{\alpha \beta}(\omega)$. In Fig.2 we display it for a frequency $\omega_{A}$ where D and F spectra of Fig.11 agree, and another frequency $\omega_{B}$ where large differences occur, and correlations must be important. Indeed a strong coupling in the subspace of states $|6\rangle,|8\rangle,|9\rangle,|10\rangle$ is observed. The corresponding wavefunctions exhibit large spatial overlap and $|6\rangle$ has the character of "bridge-state" (not shown).

In conclusion, we have shown that memory and correlation effects, predicted from a non-Markovian theory of the exciton-phonon kinetics, affect the QW absorption spectrum. Enhanced inter-peak absorption is found using realistic wavefunctions for a disordered QW, which is due to memory. Furthermore, twostep scattering events lead to correlations in the optical response. It should be possible to demonstrate these predictions by means of a QW absorption measurement, which became available recently [6].

Acknowledgements Support from DFG in the frame of Sfb 296 is gratefully acknowledged. G. M. also acknowledges support from CNR.

\section{References}

[1] P. Borri, W. Langbein, S. Schneider, U. Woggon, R. L. Sellin, D. Ouyang, and D. Bimberg, Phys. Rev. Lett. 87, 157401 (2001).

[2] P. Borri, W. Langbein, U. Woggon, V. Stavarache, D. Reuter, and A. D. Wieck, Phys. Rev. B 71, 115328 (2005).

[3] E. A. Muljarov and R. Zimmermann, Phys. Rev. Lett. 93, 237401 (2004).

[4] G. Mannarini and R. Zimmermann, Phys. Rev. B 73, 115325 (2006).

[5] G. Mannarini, R. Zimmermann, G. Kocherscheidt, and W. Langbein, phys. stat. sol.(b) 238, 494 (2003).

[6] J. R. Guest, T. H. Stievater, Xiaoqin Li, Jun Cheng, D. G. Steel, D. Gammon, D. S. Katzer, D. Park, C. Ell, A. Thränhardt, G. Khitrova, and H. M. Gibbs, Phys. Rev. B 65, 241310(R) (2002). 